\title{
COMMUNICABLE DISEASES REPORT, NEW SOUTH WALES, FOR SEPTEMBER AND OCTOBER 2006
}

For updated information, including data and facts on specific diseases, visit www.health.nsw.gov.au and click on Infectious Diseases.

\section{TRENDS}

Tables 1 and 2 and Figure 2 show reports of communicable diseases received through to the end of September and October 2006 in NSW. There were relatively few cases of arboviral infection, cryptosporidiosis and legionellosis reported in these months. Figure 2 shows reports of selected communicable diseases, by month of onset, over the past six years.

\section{SALMONELLOSIS}

\section{Outbreak of Salmonella Typhimurium PT 135a} infections

Although the total number of reports of salmonellosis declined over winter, routine surveillance detected an unseasonable increase in infection due to one strain, Salmonella Typhimurium PT 135a. From January 2006 to the end of September 2006, 108 notifications were received; the highest annual count in the past five years. Figure 1 compares the monthly count with the same month in the previous two years. Due to reporting delays the number of notifications for September is likely to be incomplete.
In response to this increase, staff from Hunter New England OzFoodNet site interviewed people notified with infection due to untyped Salmonella Typhimurium (STm) since the middle of August. These people were interviewed prior to receiving notification of the phage type so as to reduce the time between onset of illness and the date of their interview, and hence improve the quality of information provided by the interviewees.

As of the beginning of October, 42 hypothesis generating questionnaires were completed. Of these, 23 were for people who were subsequently identified as STm 135A cases. Of these 23 cases, 20 ( 87 per cent) resided in the Sydney metropolitan area, ages ranged from 2-82 years (median 13 years), and 53 per cent were female.

Information on food items consumed by cases in the seven days prior to onset were collated for each person, and information on any clusters was passed on to the NSW Food Authority for their investigation of possible sources.

The majority of cases reported consuming chicken and eggs in the seven days prior to onset of illness, although this is probably consistent with the prevalence of chicken consumption in the general community. However, 20 of 23 cases reported purchasing fresh chicken and meat from butcher shops / chicken retailers rather than from

\section{FIGURE 1}

NUMBER OF NOTIFICATIONS OF SALMONELLA TYPHIMURIUM PT 135A, NEW SOUTH WALES, FOR THE MONTHS JANUARY TO SEPTEMBER, 2004-2006

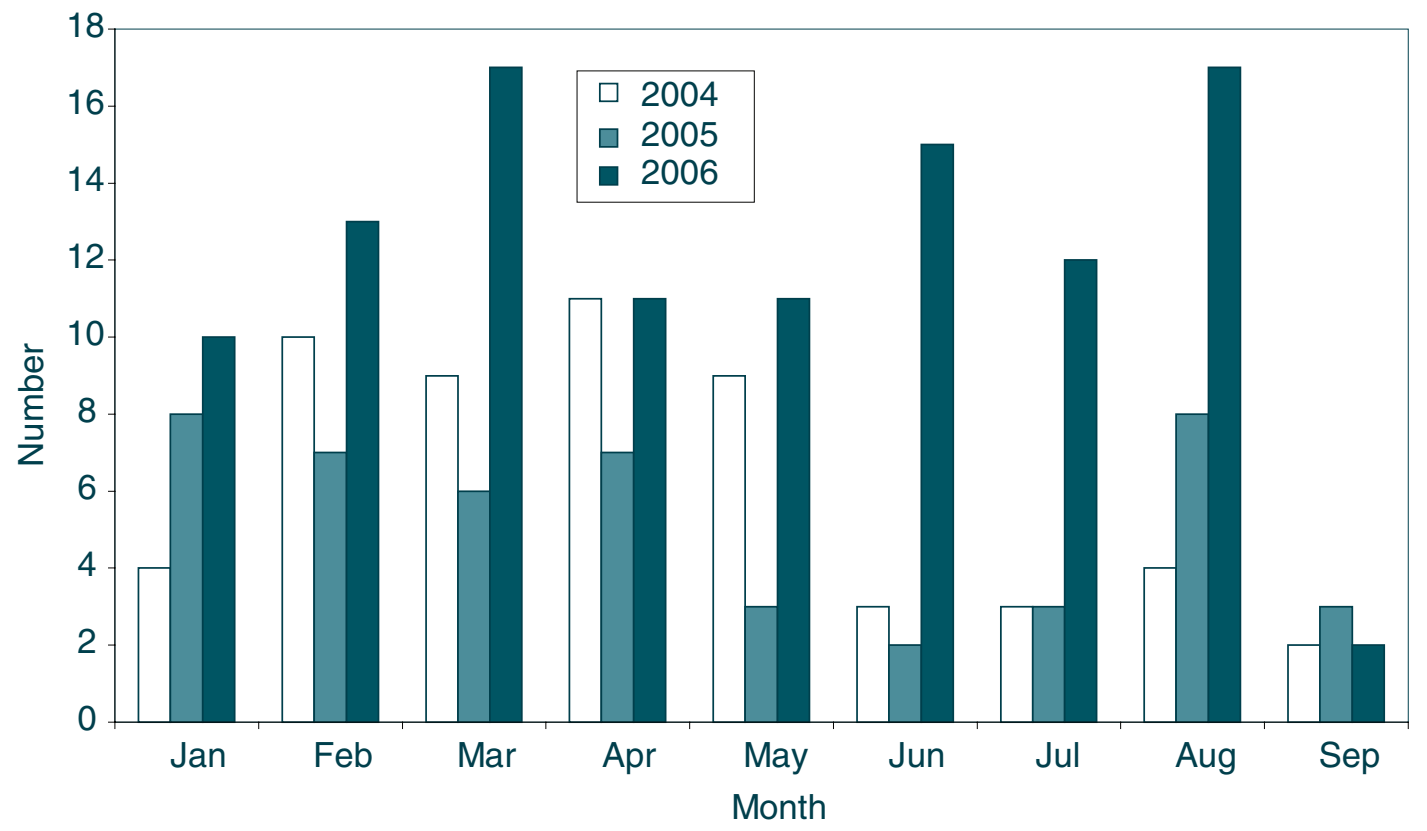


supermarkets which, in consideration of previous studies, seemed unusual. Three cases reported purchasing raw chicken products from different stores belonging to a single chicken retailing franchise.

In an environmental investigation, the NSW Food Authority found that all three shops from the implicated retail franchise sourced their fresh chicken meat from a single large poultry processor (Processor A). Subsequent testing of 30 samples from six stores of the retail franchise, including the three outlets where cases reported purchasing their chicken, found one STm135A isolate in a chicken pattie. This particular type of product had been implicated by one case. Chicken patties are made from a blend of fresh chicken meat, frozen chicken meat from spent egg layers, and a variety of spices and other ingredients. The frozen chicken meat was sourced from a Queensland company.

At the same time, whilst following up an unrelated matter, the NSW Food Authority found that a sample of raw chicken from Processor A had tested positive for STm 135A. On further investigation the NSW Food Authority found that this processor had been detecting low, intermittent levels of STm 135A in raw chicken products since January 2006. DNA fingerprinting using a technique called multiple locus variable number of tandem repeats analysis (MLVA) indicated that clinical isolates differed from those found by the NSW Food Authority on the raw chicken meat isolates. This may not necessarily exclude Processor A as the source of the outbreak.

The NSW Food Authority reported that the company implemented a number of corrective actions to minimize Salmonella contamination after being notified of the increase in human STm 135A cases. These actions included reviewing chlorine and $\mathrm{pH}$ levels in the spin chiller process, increasing staff awareness of the risk of cross contamination and the importance of personal hygiene, reviewing cleaning procedures of live bird transport systems, and thoroughly cleaning farms where STm 135A has been detected. The NSW Food Authority has continued to monitor the company and work with the poultry industry in the event of community increases of salmonellosis.

Raw meats and chicken are at risk of contamination with a range of pathogens including Salmonella. This outbreak serves as a reminder of the importance of food safety education for the consumer. Surfaces that have been in contact with raw poultry (including knives, chopping boards, containers, etc) should be thoroughly cleaned before being used to handle other products that are ready to eat. Raw poultry must be stored appropriately (refrigerated and stored separately to ready-to-eat foods) and cooked thoroughly. Finally, the importance of regular hand washing, especially after handling raw meat or poultry, must be emphasized.

\section{Outbreak of Salmonella Saintpaul infections linked to rockmelons}

In mid-October, routine Salmonella surveillance detected an increase in S. Saintpaul notifications in NSW. This serovar is relatively rare in NSW (with an average of 36 cases reported annually over the previous five years), and occurs more commonly in Queensland (with about 200 cases reported annually). In response to this increase, staff from Hunter New England OzFoodNet site conducted hypothesis-generating interviews on all new cases. Fourteen cases were interviewed and common food exposures were collated. The most startling finding was the high proportion (80 per cent) of cases that reported consuming rockmelon in the seven days prior to onset of illness.

On 24 October, a case control study was commenced to test the hypothesis that consumption of rockmelon or other foods commonly reported by the cases was associated with infection with S. Saintpaul. Subsequently reported cases and controls matched by broad age groups were asked about selected food consumed over a four-day period, place of purchase for food items and rockmelon handling and storage.

Preliminary analysis found a significant association between rockmelon consumption and infection with $S$. Saintpaul90 per cent (9 of 10) of cases reported eating rockmelon compared with 24 per cent ( 5 of 21 ) of controls. This was the only food found to be associated with illness.

Using place of purchase information obtained from the epidemiological investigation, the NSW Food Authority initiated a traceback investigation, but the exact source of the rockmelons remains unclear. A media release by NSW Health advised the public about the outbreak associated with consuming rockmelon and provided advice on rockmelon preparation and hygiene in order to reduce this risk. Advice included avoiding bruised, damaged or unrefrigerated cut rockmelons, washing hands and utensils after preparing rockmelons and refrigerating rockmelons within two hours of cutting. In addition, the NSW Food Authority advised rockmelon retailers to refrigerate melons when cut to reduce the likelihood of bacterial growth. 
FIGURE 2

\section{REPORTS OF SELECTED COMMUNICABLE DISEASES, NSW, JAN 2001 TO OCTOBER 2006, BY MONTH OF ONSET}

Preliminary data: case counts in recent months may increase because of reporting delays.

Laboratory-confirmed cases only, except for measles, meningococcal disease and pertussis $\mathrm{BFV}=$ Barmah Forest virus infections,

RRV = Ross River virus infections

Lab conf = laboratory confirmed
Men Gp C and Gp B = meningococcal disease due to serogroup $C$ and serogroup $B$ infection, other/unk = other or unknown serogroups.

NB: multiple series in graphs are stacked, except gastroenteritis outbreaks.

NB: Outbreaks are more likely to be reported by nursing homes and hospitals than by other institutions

\begin{tabular}{|cc|}
\hline \multicolumn{2}{|c|}{ NSW population } \\
Male & $50 \%$ \\
$<5$ yrs & $7 \%$ \\
$5-24$ yrs & $27 \%$ \\
$25-64$ yrs & $53 \%$ \\
$65+$ yrs & $13 \%$ \\
Rural & $46 \%$ \\
\hline
\end{tabular}

NSW population

$50 \%$

24 yrs $\quad 27 \%$

-64 yrs $53 \%$

Rural

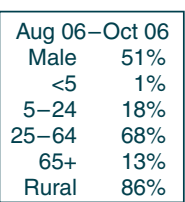

Rural $86 \%$

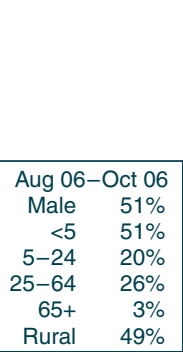

Rural $49 \%$
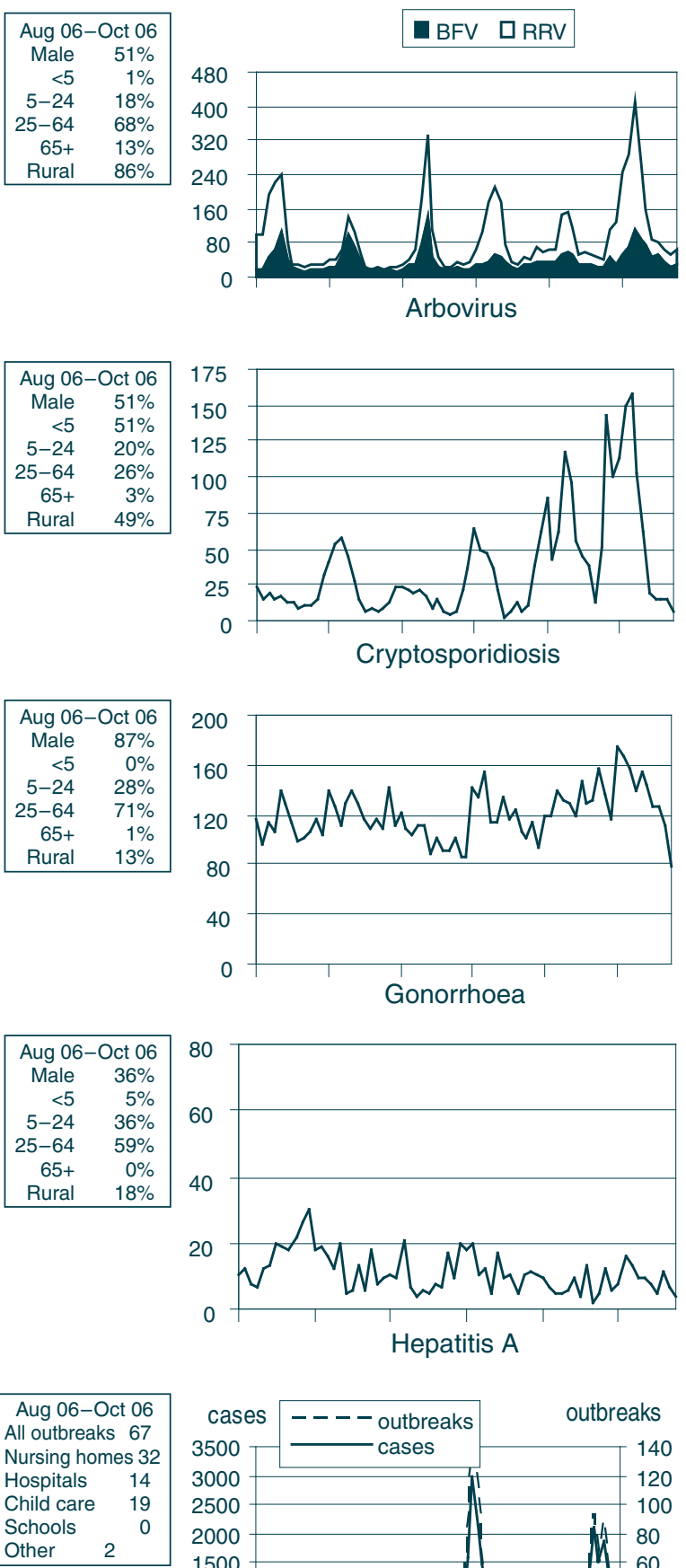

Other

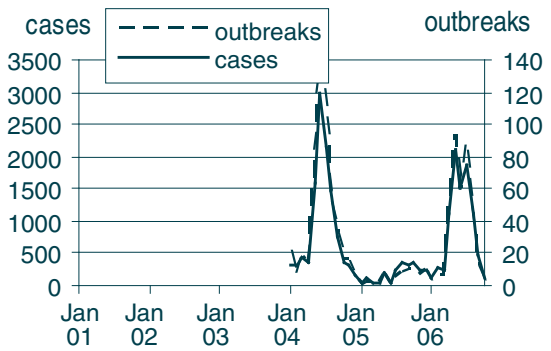

Gastroenteritis outbreaks in institutions
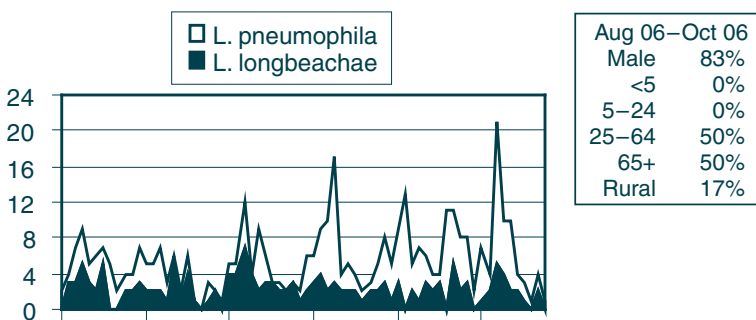

Legionellosis
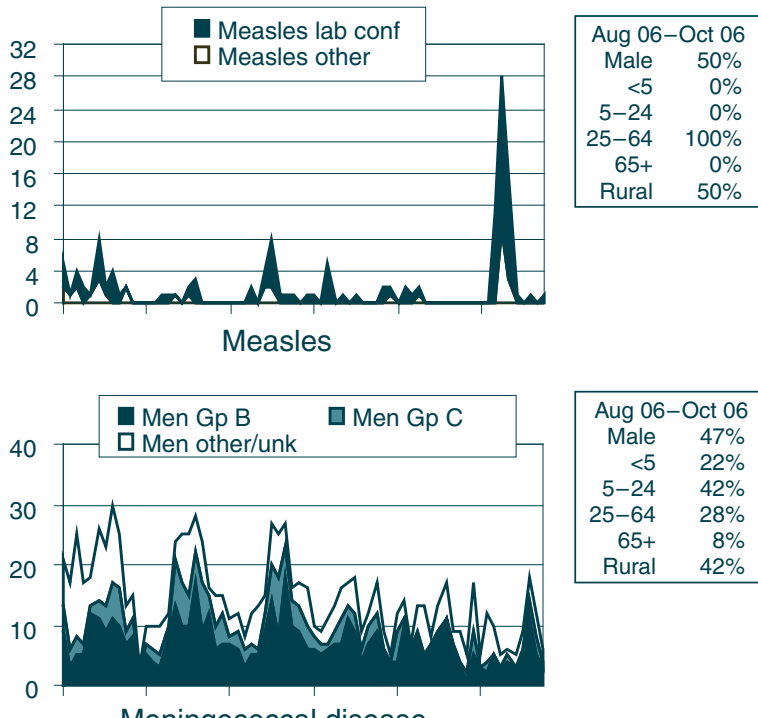

Meningococcal disease

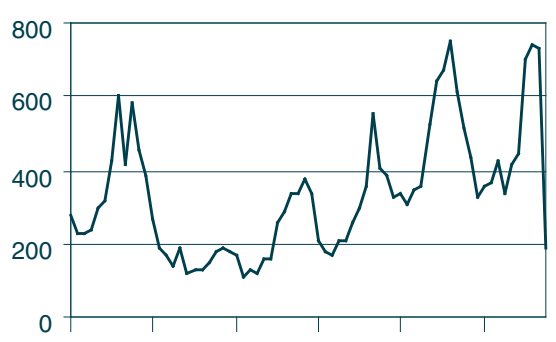

Pertussis

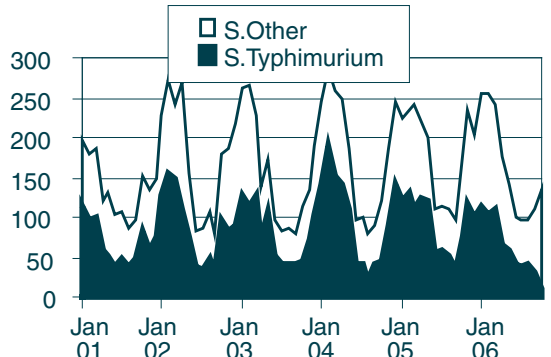

\begin{tabular}{|rr|}
\hline \multicolumn{2}{|c|}{ Aug 06-Oct 06 } \\
Male & $50 \%$ \\
$<5$ & $29 \%$ \\
$5-24$ & $27 \%$ \\
$25-64$ & $34 \%$ \\
$65+$ & $10 \%$ \\
Rural & $43 \%$ \\
\hline
\end{tabular}

Jan Jan Jan Jan Jan Jan

Salmonella infections 


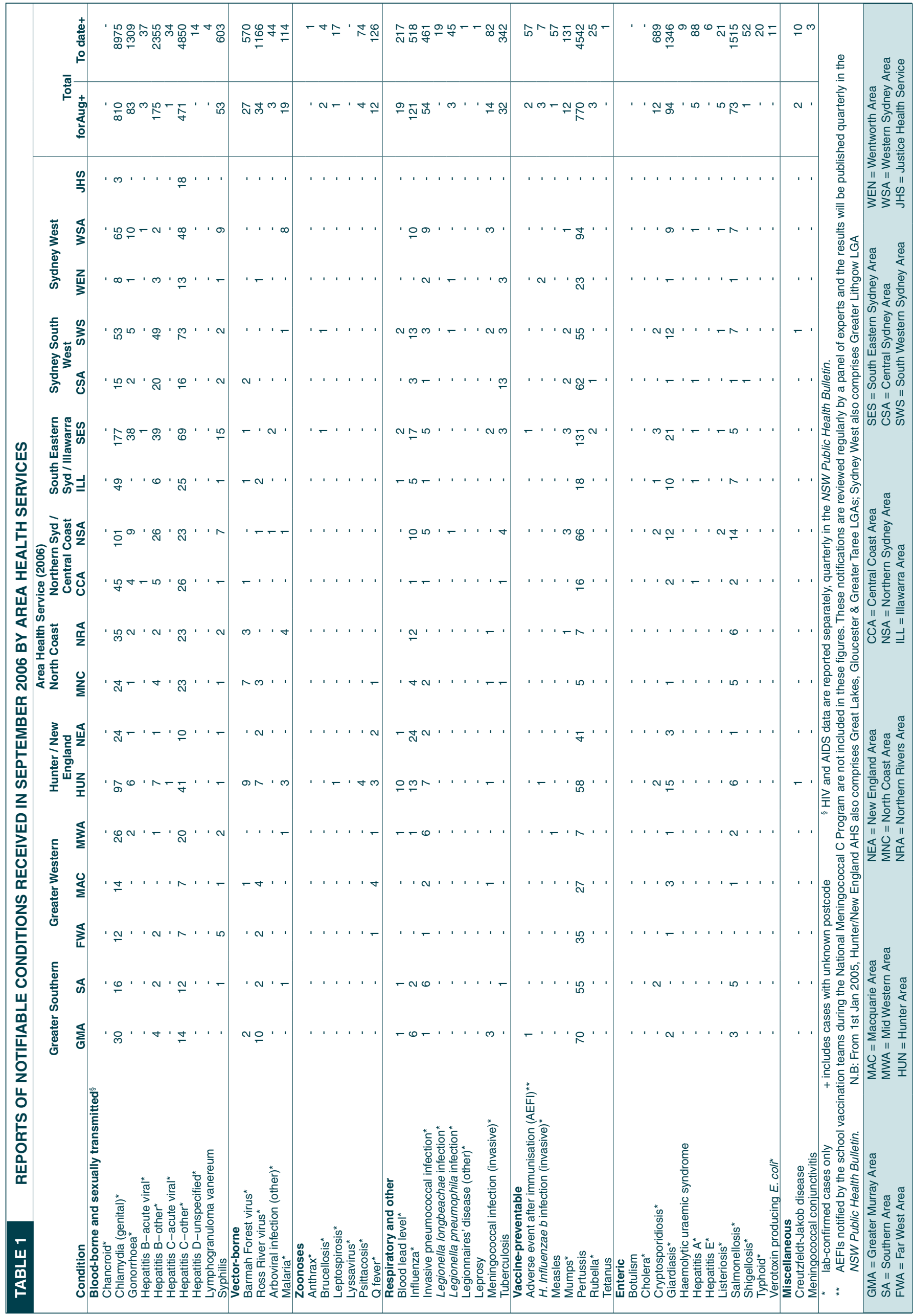




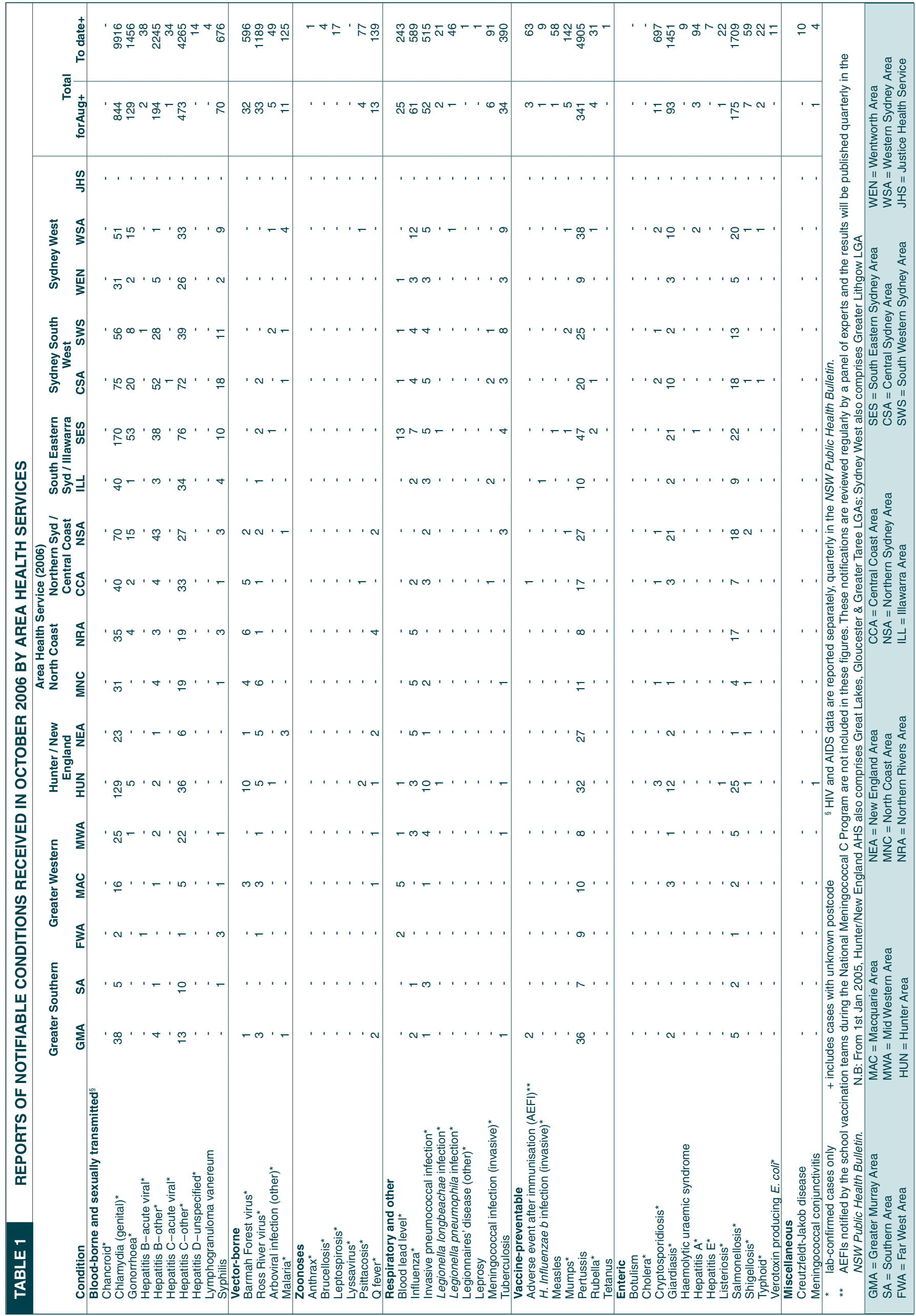

